\title{
18 „Glück“, Wissen, Politik - eine machtvolle Trias
}

Historiker^innen werden häufig gefragt, inwiefern ihre Forschungen mit der Gegenwart zusammenhängen. Aus diesem Grund schließt die Untersuchung mit einem Aktualitätsbezug. Glückswissen zirkuliert heute im jährlichen UN „World Happiness Report“, in Health Science Apps, in Fake News-Debatten, im deutschen Jugendwort des Jahres 2020 „lost“" , im deutschen Wort des Jahres 2020 „CoronaPandemie“2 sowie in den Hygge-Bewegungen während der Lockdown-Phasen zur Eindämmung der Pandemie. Hier zeigt sich die machtvolle Trias aus „Glück“, Wissen und Politik und deren Einflussnahme auf die Meinungsbildung und Stimmungslage einer Bevölkerung als ein emotionspolitisches Machtinstrument.

Der Einfluss emotionspolitischer Debatten auf die öffentliche Meinung und das Interesse seitens der Politik an der Wirkungsmacht einer affirmativen Gefühlspolitik haben in den letzten Jahren angesichts schwindender Wählerzahlen bei den sozialdemokratischen und bürgerlichen Mitteparteien zugenommen. Die Frage, wie Gefühle durch staatliche Führung gelenkt beziehungsweise unterdrückt werden können, ist jahrtausendealt und wurde seit der Antike kontrovers diskutiert (Kap. 7). Aus dem staatstheoretischen Werk Leviathan (1651) von Thomas Hobbes (1588-1679) lässt sich die Erkenntnis gewinnen, dass negative Gefühle innerhalb einer Bevölkerung (z. B. Furcht oder Misstrauen) für eine Regierung problematisch werden könnten, weil diese Gefühle einer Staatssouveränität längerfristig schaden würden. ${ }^{3}$ Für John Locke (1632-1704) war Vertrauen die Basis einer konstruktiven Beziehung zwischen der Bevölkerung und der konstitutionellen Monarchie. ${ }^{4}$ Auch heute wird die Frage nach dem Vertrauen der

1 „Das englische Wort ,lost“ wird heutzutage in der deutschen Jugendsprache in verschiedenen Situationen genutzt. Eine Person, die lost ist, ist ahnungslos, verloren oder hat einfach keinen Plan, was eigentlich gerade abgeht. Und auch dieses Wort - machen wir uns nichts vor - gibt es nicht erst seit heute [...]. Jugendwörter und Jugendsprache wandeln sich zwar ständig, werden jedoch auch durch Trends und aktuelle Themen aus den Bereichen Musik, Kunst, Gesellschaft, Politik und sogar Memes beeinflusst. Aber vielleicht passt das diesjährige Jugendwort des Jahres auch ganz gut in die aktuelle Situation [...]. Womöglich sind wir durch Corona so ,lost', wie noch nie zuvor.“ Vgl. Jugendwort des Jahres 2020: https://www.langenscheidt.com/jugendwort-des-jah res, 04.12.2020.

2 GfdS wählt ,Corona-Pandemie‘ zum Wort des Jahres 2020, Pressemittteilung vom 30.11.2020: https://gfds.de/wort-des-jahres-2020 -1/, 04.12.2020.

3 Vgl. Hobbes 1984. Vgl. Nielsen 2015, $32 \mathrm{f}$.

4 Vgl. Frevert 2013b, $160 \mathrm{ff}$.

Ә OpenAccess. (c) 2021 Isabelle Haffter, publiziert von De Gruyter. (cc))BY-NC-ND Dieses Werk ist lizenziert unter einer Creative Commons Namensnennung - Nicht kommerziell - Keine Bearbeitung 4.0 International Lizenz. https://doi.org/10.1515/9783110661439-018 
Bürger^innen in die Politik in der öffentlichen Debatte diskutiert. ${ }^{5}$ Insbesondere das zunehmend fehlende Vertrauen der Wähler*innen in den Nationalstaat ${ }^{6}$ und in die EU im Zusammenspiel mit Gefühlen des Zorns, des Hasses und des Misstrauens werden sowohl von den Medien (die selber eine Vertrauenskrise durchleben, Stichwort „Lügenpresse“) als auch von den Politikerinnen ins Zentrum der öffentlichen Aufmerksamkeit gerückt. Dies geschieht meist in der Absicht, Erklärungen für den wachsenden Erfolg rechtsextremer Bewegungen und populistischer Parteien in Europa und in den USA zu finden. ${ }^{7}$

Politik kann Gefühle lenken und umgekehrt können Gefühle die Politik manipulieren. Eine Gefühlspolitik wirkt folglich nicht nur in tribalen, antiken, monarchischen, absolutistischen, diktatorischen, totalitären oder sozialistischen Herrschaftsmodellen, sondern auch in demokratischen Staatssystemen. Eine affirmative Gefühlspolitik zur Legitimation einer Regierungsbildung spielt insbesondere in einem demokratischen Rechtsstaat eine entscheidende Rolle. ${ }^{8}$ Schließlich basiert die Legitimation einer demokratischen Regierung auf der Bereitschaft der Bürger^innen, aus moralischem Pflichtempfinden an demokratischen Wahlen teilzunehmen, das politische Geschehen im eigenen Interesse aktiv $\mathrm{zu}$ beeinflussen, um zukunftsorientiert die Politik gestalten $\mathrm{zu}$ wollen und dies auch zu können. ${ }^{9}$ Dieser freiwilligen Teilnahme an demokratischen Wahlen liegt nicht zuletzt eine, wissenshistorisch gesprochen, autosuggestiv-motivierte und selbstdisziplinarische Handlung zugrunde. Der mediale Kampf um die Stimmbürger^innen, der im jeweiligen Wahljahr zwischen den Anwärter^innen auf einen Regierungssitz ausgefochten wird, ist in den letzten Jahren immer deutlicher

5 Vgl. Allmendinger und Wetzel 2020.

6 Die Bertelsmann-Stiftung zeichnete in ihrer 2019 veröffentlichen Studie „Schwindendes Vertrauen in Politik und Parteien“ ein positiveres Bild als von den Medien dargestellt und sieht noch lange keine Gefahr für den gesellschaftlichen Zusammenhalt, vgl. Bertelsmann Stiftung 2019.

7 Vgl. Leserdiskussion „Neues Vertrauen in die EU - Wie kann das gelingen? In Rom haben die Staats- und Regierungschefs der 27 EU-Staaten eine gemeinsame Erklärung zur Zukunft des Bündnisses unterschrieben. Darin beschwören sie ihre Einigkeit und Solidarität. Doch reicht diese Botschaft aus, um die Zweifel der Bürger auszuräumen?“. In: Süddeutsche Zeitung, 25.03. 2017, vgl. Leserdiskussion 2017. Vertrauensfragen im Kontext terroristischer Bedrohungen in Berlin, vgl. Herifried Münkler, „Die Vertrauensfrage“. In: Die Zeit, 1/2017, 29.12.2016, vgl. Münkler 2016. Zu Vertrauen, Staat und Gesellschaft in der Moderne vgl. Leggewie 2011, Frevert 2013b, Frevert 2014b. Zur Frage nach einer politischen Bildung durch Gefühle vgl. Frevert 2018. Zu Hassund Zornpolitik sowie einer „Politik der Demütigung“ vgl. Emcke 2019, Jensen 2017, Frevert 2017b. 8 Vgl. Nielsen 2015, 32ff.

9 Vgl. ebd. 
zu einem Machtkampf um eine Politik affirmativer Gefühle geworden. ${ }^{10}$ Glückswissen ist ein medial wirksames Mittel, um Wählerstimmen zu mobilisieren. ${ }^{11}$ Der Erfolg der affirmativen Gefühlspolitik des ehemaligen US-Präsidenten Donald Trump („Make Amerika great again“) ist ein Beispiel.

Die machtvolle Trias aus „Glück“, Wissen und Politik ist in den letzten Jahren ein supranationales, gesellschaftspolitisches Diskursthema geworden. Eine Politik der ,Glückskulturen' steht auf der aktuellen Agenda vieler politischer Regierungsvertreter^innen, sei es in der Volksrepublik China unter dem kommunistischen Regime Xi Jinpings oder im Rahmen der „good-governance-policy“Bestrebungen $^{12}$ der $\mathrm{OECD}^{13}$, die seit 2010 einen jährlichen Bericht mit dem Titel „How is life being? Measuring Well-being “ ${ }^{14}$ veröffentlicht und darin einen ,better life index“ aller OECD-Länder (inklusive Deutschland und der Schweiz) zu bestimmen versucht.

Wie diese Messungen 2021 aufgrund der Corona-Pandemie ausfallen werden, gilt abzuwarten. Fest steht, dass weltweit Regierungsvertreter^innen und Politiker^innen, welche eine Politik der,Glückskulturen` in ihr politisches Moralsystem integriert haben, angesichts der unvorhersehbaren Folgen der Corona-Krise sowohl für die Weltwirtschaft als auch für die Lebensqualität der Weltbevölkerung vor große Herausforderungen gestellt werden.

10 Zur Politikverdrossenheit in demokratischen Ländern und zur Frage, wie aus „Wutbürgern“ „Mutbürger“ werden, um die Vertrauenskrise gegenüber demokratischen Staaten wiederzugewinnen, vgl. Streitschrift: Leggewie 2011.

11 Vgl. Hitzer und Gammerl 2013, 33, Nielsen 2015, 33f., Cabanas und Illouz 2019a.

12 Vgl. Durand M. und C. Exton (2019), „Adopting a Well-Being Approach in Central Government: Policy Mechanisms and Practical Tools“. In: Global Happiness Policy Report 2019, Substainable Development Solution Network, New York, http://happinesscouncil.org/, 15.03.2020. Auf diese Studie beruft sich u.a. die OECD in ihrem „How's Life? 2020 Measuring Well-being“-Report, https://www.oecd.org/berlin/publikationen/how-s-life-2020.htm, 20.03.2020.

13 OECD „Organisation for Economic Co-operation and Development“ („Organisation für wirtschaftliche Zusammenarbeit und Entwicklung“), Zum Hintergrund des „OECD Better Life Index“ (OECD Lebenszufriedenheit Index) heißt es erläuternd: „Die Messung von Gefühlen kann zwar subjektiv sein, ist aber dennoch hilfreich, um objektivere Daten zu ergänzen und so die Lebensqualität zwischen den einzelnen Ländern zu vergleichen. Subjektive Einschätzungen liefern Werte zu Gesundheitszustand, Bildungsniveau, Einkommen oder Selbstverwirklichung sowie zu den sozialen Bedingungen der Befragten. Umfragen werden besonders gerne genutzt, um Lebenszufriedenheit und Glücksempfindungen zu erfassen. [...] Auf einer Skala von 0 bis 10 gaben die Einwohner der OECD-Länder im Durchschnitt einen Zufriedenheitsgrad von 6.5 an.“, http:// www.oecdbetterlifeindex.org/de/topics/life-satisfaction-de/, 10.03.2020.

14 „How’s Life 2020: Measuring Well-Being“ erschien am 9. März 2020, https://www.oecd-ilib rary.org/docserver/9870c393-en.pdf?expires=1586876095\&id=id\&accname=guest\&checksum= 82FF81E3FF43F42E6020C7B30D167AE7, 10.03.2020. 
Die Soziolog*innen Eva Illouz und Edgar Cabanas betitelten ihre Studie „Happycracy“ zur Erforschung von „new coercive strategies, political decisions, management styles, consumption patterns, individual obsessions and hierarchies that, together with a new notion of citizenship, have emerged in the age of happiness. " ${ }^{15}$ Zum Beweis ihrer pointiert formulierten Thesen über eine Glückstechnokratie („Techno-Happycracy“) ${ }^{16}$ nennen die Soziolog^innen die seit einigen Jahren boomende Positive Psychology und die damit im Zusammenhang stehende Happiness Economy. ${ }^{17}$

Die empirische Erkenntnis über die Konstruiertheit arbeitspsychologischen Glückswissens, welche Illouz und Canabas in ihrer gesellschaftskritischen Studie zutage fördern, ist für unsere Gegenwart aufschlussreich. Dennoch sind das Phänomen und die Praktik einer arbeitspsychologischen Instrumentalisierungsstrategie einer Politik der ,Glückskulturen', aus einer wissenshistorischen Perspektive betrachtet, nicht „new“. Die vorliegende historische Untersuchung hat im Übergang von der Weimarer Republik zur NS-Zeit im transnationalen Vergleich mit der Schweiz gezeigt, dass Autosuggestion, Selbstoptimierung und Leistungsorientiertheit einem ambivalenten Glückswissen zugrunde liegen, um politische und wirtschaftliche Interessen zu verschleiern oder diese $\mathrm{zu}$ festigen.

Ein weiterer Aktualitätsbezug der vorliegenden historischen Untersuchung über eine Politik der ,Glückskulturen' geht aus dem Wort des Jahres 2016 hervor: „Postfaktisch“. Die „Gesellschaft für Deutsche Sprache“ (GfdS) begründete ihre Wahl für das Wort des Jahres 2016 damit, dass sie den Fokus auf einen aktuell „tiefgreifenden politischen Wandel“ richten wollte. ${ }^{18}$ Aus Sicht einer Historikerin mangelt es dieser Behauptung jedoch an emotions- und wissenshistorischer Präzision. Wie die vorliegende historische Untersuchung zu zeigen versucht, befassten sich Politiker*innen, Wissenschaftler`innen und Kunstschaffende spätestens seit der Antike in unterschiedlichen, sich zeitlich wandelnden und in sich widersprüchlichen Wissensdisziplinen mit der Bedeutung und Funktion der Trias aus „Glück“, Wissen und Politik und deren wechselseitiger Einflussnahme auf die Meinungsbildung und Stimmungslage einer Bevölkerung als ein massenpsychologisches Machtinstrument.

15 Cabanas und Illouz 2019b, 33. Originaltitel: „Happycracy, Comment l'industrie du bonheur a pris le contrôle de nos vies“, vgl. Cabanas und Illouz 2018.

16 Vgl. Cabanas und Illouz 2019b, 43.

17 Prominente Beispiele sind der „Oxford Happiness Inventory (OHI)“ der „Satisfaction With Life Scale (SWLS)“, der „PANAS (Positive Affect, Negative Affect Schedule)“, die „Experience Sampling Method (ESM)“ oder die „Day Reconstruction Method (DRM)“, vgl. Cabanas und Illouz 2019b, 70 f. 18 Vgl. Doer und Mikö 2016. 
Wie wirkungsmächtig die Deutungshoheit von „Glück“ im Untersuchungszeitraum 1933-1945 war und in welchen emotionspolitischen Praktiken und an welchen Orten Gefühlswissen ambivalent angewandt, verändert, verhandelt, zurückgewiesen und umgedeutet wurde, hat die transnational und vergleichend angelegte Untersuchung exemplarisch aufzuzeigen versucht. Das vorliegende Buch nimmt die Begrifflichkeit der Politik der,Glückskulturen` daher auch in ihrer Pluralform auf, um sie erstmals im Zeitraum 1933-1945 anhand von zwei deutschsprachigen Nachbarländern mit unterschiedlichen politischen Systemen zu untersuchen: die demokratische Schweiz auf der einen Seite und die totalitäre NS-Diktatur Deutschlands auf der anderen Seite, um Brüche, Ambivalenzen und Kontinuitäten aufzudecken. 
